\title{
Commentary
}

\section{Domestic violence: an emerging concern in maternity care}

\author{
Lakshmen Senanayake ${ }^{1}$ \\ Sri Lanka Journal of Obstetrics and Gynaecology 2011; 33: 142-149
}

\begin{abstract}
Domestic violence, a malady cross cutting all strata of the society, affects mostly women, but not exclusively, and affects their health and well being, particularly in the area of reproductive health. Pregnant women undergoing violence are at a higher risk of experiencing many negative health outcomes affecting the mother-to-be and the unborn baby. They include miscarriages, unwanted pregnancies leading to unsafe abortions, fetal death, growth restriction, abruption of the placenta and even homicide by the partner. Violence limits the freedom of making contraceptive choices for the woman making her vulnerable to uncontrolled fertility. Few women would volunteer information about abuse and unless the care provider is alert and vigilant the opportunity to identify and support her to prevent further violence will be lost. Many professional bodies recommend 'routine inquiry' from all pregnant women. It is also important to be on the lookout for indicators of domestic violence among pregnant women as they have many barriers preventing them from disclosing violence. Providing emotional support is an important component of care which is likely to prevent post partum depression and other mental ill health outcomes which are common among women undergoing domestic violence. It is important to recognize that women undergoing violence in pregnancy needs special attention and the care providers should build their capacity to care for them.
\end{abstract}

\section{Introduction}

Domestic violence, often inflicted by the intimate partner, occurs in all countries and communities, irrespective of social, economic, religious, or cultural identities. Although women can be violent in

\footnotetext{
${ }^{1}$ Consultant Obstetrician and Gynaecologist

Correspondence: Lakshmen Senanayake

E-mail: laksena@hotmail.com
}

relationships with men, the overwhelming burden of domestic violence is borne by women at the hands of men ${ }^{1}$.

Domestic violence is gradually being acknowledged as one of the most severe threats to women's health and pregnant women comprise an important segment of this larger group.

In contrast to the common belief, domestic violence is seen frequently in pregnancy, often starting or escalating during pregnancy $y^{2,3}$.

Although data on domestic violence came to be available over the last few decades, impact of domestic violence in pregnancy came to be critically evaluated and assessed only in the recent past.

Domestic violence in pregnancy has emerged as a national and a global health issue, which has the potential to produce serious negative health impacts, sometimes fatal, to the mothers-to-be, and to the unborn fetus. Harmful consequences of domestic violence extend beyond the birth affecting the mother and the newborn.

It is well established that domestic violence contributes to many pregnancy complications such as miscarriage, ante partum haemorrhage, premature labour and generates negative health behaviours from women, such as late registration and inconsistent attendance for antenatal care which in turn increase her risk status.

It is a tragic situation that this topic is often considered by many care providers as shameful, irrelevant, leading to unnecessary intervention. Such attitudes leave the abused woman uncomfortable to tell the provider and the health care provider uncomfortable to ask, and the health care system is not able to utilize the window of opportunity offered during pregnancy, to identify and care for the abused woman.

Inadequacy of health-care workers' knowledge and the societal constrains that affect their attitudes towards the subject, and the ensuing reluctance to "open the Pandora's box" makes it's detection and 
management inconsistent, ineffective and sometimes counterproductive.

Against this depressing background, it is encouraging to recognize that most professional associations such as The Royal College of Obstetricians and Gynecologists of UK, American College of Obstetrics and Gynecology have recommended that identification of abused women and their needs be conducted as a part of routine antenatal care.

This subject has been addressed as a separate annexure in the last three reports of the Confidential Inquiry in to Maternal Deaths UK indicating the importance and relevance of this subject in maternity care $^{4}$.

\section{What is domestic violence?}

Domestic violence, in general terms, has been commonly referred to as physical, sexual or psychological violence against a woman perpetrated by a current or former partner ${ }^{5}$.

Domestic violence is defined as "any incident of threatening behavior or abuse (psychological, physical, sexual, financial or emotional) between adults who are, or have been intimate partners or family members, regardless of gender or sexuality" 6 .

The Center for Disease Control USA, defines domestic violence during pregnancy as "Physical, sexual, or psychological/emotional violence, or threats of physical or sexual violence that are inflicted on a pregnant woman"7.

Domestic violence often follows a systematic and sustained pattern of abusive and coercive behaviors, occurring within a relationship, regardless of race, ethnicity, age, income, religion, education or sexuality. This commonly occur at home, and often perpetrated by someone who carries a "protective aura" such as husband, boy friend, father or mother in law.

The Prevention of Domestic Violence Act 2005 Sri Lanka, which is gender neutral, defines domestic violence, inclusive of emotional violence, in a broad manner and includes violence between two members of the family including children and the elderly ${ }^{8}$.

\section{Prevalence of domestic violence in pregnancy}

Globally, domestic violence is the most common form of violence affecting women, in contrast to forms of violence affecting men, often perpetrated by the intimate partner and being pregnant is no exception.
Unfortunately, bulk of published work on domestic violence, to date, has focused on women in general and not specifically on pregnant women. Available information on domestic violence on pregnant women, mostly come from developed countries and often use facility-based data'.

There is considerable variation of prevalence of domestic violence in pregnancy, in different studies. This is largely due to differences in the definitions of violence used, when and how many times women have been asked during the pregnancy and the populations studied.

The Multi Country Study by WHO, covering 10 countries and 24,000 women, found that the proportion of pregnant women, who reported (only) physical violence, during, at least one pregnancy, varied considerably, from $1 \%$ in Japan to $28 \%$ in Peru, with most of the countries falling between $4 \%$ and $12 \%^{10}$.

The World Report on Violence records rates, ranging from $6 \%$ to $15 \%$, of ever partnered women who have been physically or sexually abused in pregnancy usually by the partners ${ }^{1}$.

$4 \%$ of child bearing women reported physical violence by husband or partner around the time of pregnancy to the Pregnancy Risk Assessment Monitoring System (PRAMS) of the USA ${ }^{11}$.

It is estimated that in USA as many as 324,000 women experience intimate partner violence during their pregnancy, each year ${ }^{12}$.

$23.5 \%$ of pregnant mothers in UK reported a lifetime history of domestic violence and 3\% of them reported being violated during the current pregnancy, which occurred everyday or nearly every day ${ }^{5}$.

A longitudinal cohort study from Australia among pregnant women, targeting, enrolment, 6 and 12 months post partum recorded that $17 \%$ of women experienced physical and / or emotional abuse ${ }^{13}$. Study from New Zealand found that six per cent of urban women and $9 \%$ of rural women had ever experienced violence during pregnancy with approximately $40 \%$ of them had experienced violence in more than one pregnancy ${ }^{14}$. A study from a family practice clinic from Canada recorded an, overall prevalence rate of domestic violence in pregnant women as $14.6 \%$. emotional abuse was reported by $10.4 \%$, threat of violence by $8.3 \%$, and physical or sexual violence by $7.6 \%^{15}$. The trust and confidence in the family practitioner may be the reason for higher reporting. 
Significantly higher rates of domestic violence were recorded from Pakistan where $51 \%$ of women reported experiencing verbal, physical or sexual abuse in the six months prior to, and/or during pregnancy with $16 \%$ percent of women considering suicide as a response to the abuse ${ }^{16}$.

Similar experience was seen in a prospective cohort study from Brazil, of pregnant women attending primary health-care clinics with $28 \%$ of them reporting emotional abuse ${ }^{17}$.

A study of domestic violence in a cohort of 1200 pregnant women in Badulla Sri Lanka, found that $4.7 \%$ of them were physically abused during the current pregnancy with $2.7 \%$ experiencing sexual violence. It was interesting to note that the perpetrator identified was the husband in $60 \%$ of women and mother in law in $17 \%{ }^{18}$.

In this context it could be considered that domestic violence may be more common than some of the conditions for which mothers are routinely screened for ${ }^{19}$.

\section{Are pregnant women at a greater risk of DV?}

Some women are first abused during pregnancy, while for others, the violence is a part of an ongoing pattern of systematic abuse ${ }^{1}$, and, pregnancy does not stop or lessen the beatings.

In more than $30 \%$ of women abused in pregnancy violence first starts during the pregnancy ${ }^{3}$, and the risk of pregnant women's risk of abusive violence is shown to be $60.6 \%$ greater than that of non pregnant women ${ }^{20}$. However the risk seems to differ between countries with Ethiopia recording 13\% and Brazil, Serbia and Montenegro recording 50\% ${ }^{10}$. Cultural attitudes towards motherhood may be a possible explanation.

For the most recent pregnancy in which women had experienced violence, most was perpetrated by the child's biological father $(96 \%)^{14}$.

Women are four times more likely to suffer increased abuse as a result of an unintended or unwanted pregnancy. Such pregnancies themselves could be the results of domestic violence, in the form of sexual abuse, marital rape or denial of access to birth control ${ }^{1,21}$.

A study from Pakistan, revealed that young maternal age, having an unemployed husband and one with other wives/partners, and having had a prior pregnancy were significant predictors of abuse in pregnancy $^{16}$.
Pregnant adolescents in particular, have an elevated risk of violence from their partners ${ }^{4,22}$. Common reason given for the increased risk of violence during pregnancy is that the father/male partner feels a greater sense of stress over the impending birth. The stress manifests itself as frustration, which is directed back at the perceived source: the mother and her unborn child.

Pregnancy is one of those situations where the woman gets more attention. Psychologically, some perpetrators perceive this as a threat to their dominance. Domestic violence during pregnancy is a focused attack that puts not just one, but two lives at risk. Unlike in non pregnant women undergoing domestic violence, where the head is usually attacked, battering of pregnant women tend to be directed at abdomen, breasts, or genitals ${ }^{23}$.

\section{What are the consequences of domestic violence in pregnancy?}

Evidence collected over the last 20 years indicates that domestic violence during pregnancy poses a threat to health, both short- and long-term, and at its extreme, can result in the death of the mother and / or her unborn baby. This is important in the South Asian context where both fertility and domestic violence are high but is poorly understood ${ }^{24}$.

Adverse outcomes in pregnancies with domestic violence may occur directly as in placental separation due to battering or indirectly as a result of associated factors such as late entry to antenatal care, frequently repeated pregnancies, lack of social support, and tendency to follow risky behaviors such as smoking and alcohol abuse.

In addition, depressive symptoms too will influence the mother's perceptions, tolerance and the responses to onset of symptoms and health needs that might potentially jeopardize her health. The general loss of interest on the part of the mother, in her or her baby's health, both during the pregnancy and after the child is born is a matter for major concern $^{5,14,25,26}$.

\section{Miscarriages}

Likelihood of an unwanted pregnancy is high in a relationship with domestic violence due to lack of decision making opportunities for the woman in use of contraception. A study recorded that women who had experienced violence in pregnancy, compared with those who had not, were less likely to report that the pregnancy was wanted at that time (28\% vs $55 \%)$, 
and less likely to report that their partner wanted the pregnancy $(40 \% \text { vs } 57 \%)^{14}$.

Domestic violence may lead to induced abortions as she may be compelled to terminate the pregnancy because it is unwanted or is a result of forced sex or she may be pressurized by her partners or family members. On the other hand women who have opted for an abortion may suffer violence in retaliation ${ }^{27}$.

The WHO study and other investigators have found a significant association between domestic violence and pregnancy losses such as miscarriages, still births and neonatal deaths ${ }^{10,28}$.

\section{Fetal death}

A study from Canada and another from India did find a strong association of fetal death and domestic violence with the Canadian study recording a seven times risk of peri natal deaths in abused women compared to non abused women ${ }^{29,30}$. An Indian study also showed a significant increase. Systematic review of the literature revealed that domestic violence is responsible for increased fetal deaths in affected pregnancies to 16 per $1000^{31}$.

\section{Homicide}

Homicide is a leading cause of traumatic death for pregnant and postpartum women in the United States. Evidence exists that a significant proportion of female homicide victims are killed by their intimate partners $^{32}$.

The UK Confidential Inquiry 2006-2008 found 55 coincidental deaths of which 11 deaths were homicides and seven of them were killed by their partners and recommends not to consider women who suffer from DV as low risk ${ }^{4}$.

The World report on violence too draws attention to the substantial but largely unrecognized proportion of women, murdered by the partner in Bangladesh and India ${ }^{1}$.

\section{Low birth weight}

Many studies have explored the incidence of low birth weight in pregnancies of abused women but found mixed results. Interesting finding from USA showed women from the private hospitals who suffered from DV had a four times higher chance of getting a low birth weight baby than those who were not abused. This risk was not seen in women who attended state health care $^{33,28}$.
Same finding was seen in a meta analysis of eight studies which showed a slight but statistically significant difference of birth weight of babies born to abused and non abused women ${ }^{34}$.

\section{Preterm labour}

Association of preterm labor and domestic violence has been recorded in few studies ${ }^{28,29}$. One of the studies noted that while the incidence of preterm labour was $6.9 \%-10 \%$ in the general population while that of the study population was $15.4 \%$ for moderately abused women and $17.2 \%$ for severely abused ${ }^{30}$.

\section{APH}

A study from Canada on women abused during pregnancy had 3.5 higher odds for ante partum hemorrhage than those not abused ${ }^{30}$.

\section{Depression}

Psychological violence during pregnancy by an intimate partner is strongly associated with postnatal depression, independent of physical or sexual violence. The most common form of partner violence was psychological and its frequency was positively associated with occurrence of postnatal depression ${ }^{17}$. A prospective cohort study on post partum depression found that of the $16 \%$ of women who had depressive symptoms, $40 \%$ reported domestic violence $^{35}$.

\section{Contraception}

Violent men typically seek control over their partner's behavior and sexuality and women are often unable to negotiate the timing of pregnancy by use of contraception. Some abused women got pregnant to please their partners, while others are forced into abortions against their will. Very often the freedom to use contraception is limited in a relationship with violence. A recent study in Bolivia found that of 300 women interviewed, 5 percent were physically abused and 15 percent verbally abused by their partners because of their contraceptive use. And other studies report that women are even afraid to bring up the subject of contraception because of possible retribution from their partners ${ }^{36}$.

For these women adequate and patient counseling is necessary while recognizing the woman's difficulties with her partner and help her to choose the method that will not make those difficulties worse. 


\section{Direct effects of physical trauma in pregnancy}

When a woman is physically battered particularly on the abdomen which is a common target, complications may directly result leading to premature rupture of membranes and placental abruption. A study from USA of pregnancies complicated by trauma found that domestic violence was responsible for $22.2 \%$ of the trauma admissions of pregnant women of which $40 \%$ of women had painful contractions resulting in $11 \%$ going in to labour and $2 \%$ of women experiencing an abruption of the placenta $^{37}$.

\section{Screening for DV in pregnancy in maternity care}

Most women undergoing abuse will not disclose the fact spontaneously, unless they are inquired about violence.

Many studies have illustrated that pregnant women accept and find no offense in being screened for DV by their healthcare providers. When screening questions were administered as a pilot study in Anuradhapura, $96 \%$ of women agreed to respond to the screening questions ${ }^{38}$.

Women are often reluctant to disclose abuse or violence to their healthcare provider for fear that it will be reported or disclosed.

Apart from screening of mothers done for research purposes universal screening is not practiced in most countries in the region ${ }^{39}$.

The UK National Screening Committee does not recommend universal screening of all mothers for domestic violence in pregnancy as a policy ${ }^{40}$. This is different from routine inquiry as recommended by many organizations.

RCOG guideline on antenatal care, emphasizes the importance of identifying women undergoing abuse and recognizing negative health impacts and makes a category $\mathrm{D}$ recommendation which states "Healthcare professionals need to be alert to the symptoms or signs of domestic violence and women should be given the opportunity to disclose domestic violence in an environment in which they feel secure $^{\prime 41}$

Confidential Inquiry in to maternal deaths comments that "Inquiry continues to recommend that routine enquiry, 'Asking the question', should be made, about domestic abuse, either when taking a social history at booking or at another opportune point during a woman's antenatal period. The antenatal booking appointment may be the appropriate time to 'ask the question' or the midwife may decide to delay until the following appointment when a relationship has already been established" 4 . The report highlights the need for appropriate documentation and the availability of referral and support mechanisms as a prerequisite.

The American College of Obstetricians and Gynecologists (ACOG) and the Center for Disease Control and Prevention (CDC) USA recommend that routine screening should occur at various times over the course of the pregnancy. Screening should be at the first prenatal visit, at least once during each trimester, and at the postpartum visit ${ }^{42}$.

In spite of the above recommendations a study from USA found that $56 \%$ of respondents including obstetricians reported that $50.5 \%$ rarely or never screen their female patients for domestic violence; and 52\% reported their competence for providing treatment for victims as poor to fair ${ }^{43}$.

Although few authors from the developing countries recommend universal screening ${ }^{9}$ most countries with heavy antenatal care services face constraints in providing time, privacy, skilled personnel, protocols and lack a mechanism to effectively respond. Therefore universal screening is not acceptable for most countries, ${ }^{39}$ but routine inquiry as practiced in UK should be considered mandatory.

\section{Responding to domestic violence}

It is important to recognize that both care provider and the receiver have to overcome many challenges in order to start communicating on the issue of domestic violence. Barriers patient has to face include fear of escalating violence, fear of exposure to third parties, fear of being blamed or ridiculed and reporting to police. The provider constraints include lack of time, priority issues, lack of knowledge and skill to respond and lack of supportive mechanism to help when the problem is identified. Disclosure is the result of a dialogue built on trust and confidence. It is essential to adhere to important guiding principles such as maintaining privacy, confidentiality, safety, non discrimination and treating women with respect in order to create a trusting environment that enables the discussion of sensitive and difficult matters ${ }^{43}$.

Very few women volunteer information and many would deny it initially, and it is important to be on the lookout for indicators suggestive of domestic violence. None of these automatically indicate abuse but they should raise suspicion and prompt one to make further inquiry. 
Table 1. Indicators of domestic violence

Indicators of domestic abuse, relevant to maternity care

(Report of the Confidential Enquiries into Maternal Deaths in the United Kingdom 2006-2008²)

- Late booking and/or poor attendance or non attendance at antenatal clinics

- Repeat attendance at antenatal clinics, the GP surgery or Emergency Departments for minor injuries or trivial or nonexistent complaints

- Unexplained admissions

- Noncompliance with treatment regimens/early self discharge from hospital

- Repeat presentation with depression, anxiety, self-harm and psychosomatic symptoms

- Injuries that are unattended and of several different ages, especially to the neck, head, breasts, abdomen and genitals

- Minimalisation of signs of abuse on the body

- Sexually transmitted diseases and frequent vaginal or urinary tract infections and pelvic pain

- Poor obstetric history:

- repeated miscarriage or terminations of pregnancy

- stillbirth, or preterm labour

- preterm birth, intrauterine growth restriction/low birthweight

- unwanted or unplanned pregnancy

- The constant presence of the partner at examinations, who may be domineering, answer all the questions for her and be unwilling to leave the room

- The woman appears evasive or reluctant to speak or disagree in front of her partner.

The recently delivered report of the Taskforce on the Health Aspects of Violence Against Women and Children, UK iterated that "Central to the capability required of all NHS staff is the ability to understand the risk factors for, and recognize the signs of, violence and abuse - not all of which are obvious. Clinicians should be more open to the possibility that violence or abuse is an underlying cause of the problems of the patient in front of them. But this recognition in itself is not enough - women and children told us that staff needs to build trust, demonstrate belief in what they are told and discuss options sensitively. This means that practitioners must develop the communication skills (both verbal and non-verbal)" ${ }^{3}$.

Providing emotional support to the survivor is an important aspect of caring. Although it is difficult to achieve the skills of a professional counselor by all care providers, they need to develop listening and befriending skills. Principles of befriending include getting to know each other, bonding and building a relationship, empathetic but active listening and using appropriate body language.
Documentation and data management is one of the most important but often neglected areas of care provision $^{39}$. Documentation necessitates a clear, precise and comprehensive record of the history taken of the incident as well as a description of the injuries observed preferably on body maps. This is important even when the survivor has not opted to inform police or take a legal remedy.

Supporting the woman undergoing violence needs a multi sector response. It is important for the providers of maternity care to building up partnerships and linkages with relevant parties.

\section{Conclusion}

Until recently most governments, policy makers and health care providers considered domestic violence as a minor social issue, affecting few women which needs to be addressed essentially by the legal sector. Research and information generated at many academic discussions, international conventions and forums have produced undeniable evidence that has 
convinced the skeptics, that domestic violence is a major public health issue and pregnant women undergoing abuse are at a high risk of multiple negative health outcomes, affecting both mother and the baby. Health care providers have a role and a responsibility to design strategies to identify them and provide effective, sensitive and holistic care to pregnant women living with abuse.

\section{References}

1. World Report on Violence and Health . Published by the World Health Organisation, Geneva, 2002. ISBN 9241545615.

2. Zero tolerance for domestic violence. The Lancet 2004; 364: 1556.

3. Responding to violence against women and children - the role of the NHS. The Report of the Taskforce on the Health Aspects of Violence Against Women and Children, March 2010.

http://www.health.org.uk/media_manager/public/75/ external-publications / Responding-to-violence-againstwomen-and-children $\%$ e $\% 80 \% 93$ the-role-of-the-NHS.pdf

4. Saving Mothers' lives Reviewing maternal deaths to make motherhood safer: 2006-2008: British Journal of Obstetrics and Gynaecology 2011; 118 (Supplement 1).

5. Bacchus L, Mezey G, Bewley S. Domestic violence: prevalence in pregnant women and associations with physical and psychological health. European Journal of Obstetrics and Gynecology and Reproductive Biology 2004; 113: 6-11.

6. Responding to Domestic Abuse. A Handbook for Health Professionals. Department of Health London, 2006. www.dh.gov.uk/publications.

7. Domestic violence during pregnancy. Fact Sheet. Women development and health programme. Pan American Health Organisation (PAHO), WHO.

8. Prevention of Domestic Violence Act, No.34 of 2005. Published as a Supplement to Part 11 of the Gazette of the Democratic Socialist Republic of Sri Lanka of October 07, 2005. http://webapps01.un.org/vawdatabase/uploads/ Sri\%20Lanka\%20-\%20Prevention\%20of\%20Domestic\%20 Violence\%20Act\%20(2005).pdf

9. Koski AD, Stephenson R, Koenig MR. Physical violence by partner during pregnancy and use of prenatal care in rural India. Journal of Health Population and Nutition 2011; 29(3): 245-54.

10. Moreno CG, Henrica AF, Jansen MM, Ellisberg M, Heise L, Watts. WHO Multi-country Study on Women's Health and Domestic Violence: Initial Results on Prevalence, Health Outcomes and Women's Responses. Published by WHO Press, Geneva, 2005: 63-72.

11. Domestic Violence and Pregnancy, Facts For Health Care Providers: Washington State Department of Health, August 2008. www.wscadv.org

12. Gazmararian JA, Petersen R, Spitz AM, Goodwin MM, James S. Violence and reproductive health: Current knowledge and future research directions. Maternal and Child Health Journal 2000; 4.
13. Gartland D, Hemphill SA, Hegarty K, Brown SJ. Intimate partner violence during pregnancy and the first year postpartum in an Australian pregnancy cohort study. Maternal and Child Health Journal 2011; 15(5): 570-8.

14. Fanslow J, Silva M, Robinson E, Whitehead A. Violence during pregnancy: associations with pregnancy intendedness, pregnancy-related care, and alcohol and tobacco use among a representative sample of New Zealand women. Australian NZ Journal of Obstetrics and Gynaecology 2008; 48(4): 398-404.

15. Ahmad F, Hogg-Johnson S, Stewart DE, Levinson W. Violence involving intimate partners: prevalence in Canadian family practice. Canadian Family Physician 2007; 53(3): 4618,460 .

16. Karmaliani R, Irfan F, Bann CM, McClure EM, Moss N, Pasha O, Goldenberg RL. Domestic violence prior to and during pregnancy among Pakistani women. Acta Obstet Gynecol Scand 2008; 87(11): 1194-201.

17. Ludermir AB, Lewis G, Valongueiro SA, de Araajo TV, Araya R. Violence against women by their intimate partner during pregnancy and postnatal depression: a prospective cohort study. Lancet 2010; 376(9744): 903-10. Epub 2010 Sep 6.

18. Moonesinghe L, et al. Development of a screening instrument to detect physical abuse and its use in a cohort of pregnant women in Sri Lanka. Asia Pacific Journal of Public Health 2004; 16(2): 138-44.

19. Intimate partner violence in pregnancy. A Guide to Clinicians developed by ACOG and CDC.

20. Newberger, Eli $\mathrm{H}$, et al. Abuse of pregnant women and adverse birth outcome. Midwifery Today 1993; Issue 26, http://www.midwiferytoday.com/reviews/abuse.asp

21. Heise L. Reproductive freedom and violence against women: what are the intersections? The Journal of Law, Medicine and Ethics 1993; 27. http://onlinelibrary.wiley.com/doi/ 10.1111/j.1748-20X.1993.tb01243.x/abstract

22. Family Planning. Perspectives 1999; 31.

23. Bewley C. Coping with domestic violence during pregnancy. Nursing Standard 1994; 8.

24. Koski AD, Stephenson R, Koenig MR. Physical violence during pregnancy and use of prenatal care in India. Journal of Health Population and Nutrition 2011; 29(3): 245-54.

25. Violence against women. Relevance for medical practitioners. Council on Scientific Affairs, American Medical Association. Journal of American Medical Association 1992; 267(23): 3184-9.

26. Newberger EH, Barkan SE, Lieberman ES, McCormick MC, Yllo K, Gary LT, Schechter S. Abuse of pregnant women and adverse birth outcome. Current knowledge and implications for practice. Journal of American Medical Association 1992; 267(17): 2370-2.

27. de Bruyn M. Violence, pregnancy and abortion: issues of women's rights and public health. A review of worldwide data and recommendations for action IPAS, 2003.

28. Sarkar NN. The impact of intimate partner violence on women's reproductive health and pregnancy outcome. Journal of Obstetrics and Gynaecology 2008; 28(3): 266-71. 
29. Pallittio CC. Domestic violence and maternal, infant and reproductive health: a critical review of the literature. Submitted to Pan American Health Organisation (PAHO) Washington, 2004. http://www.docstoc.com/docs/ 40362692/ Domestic-violence-is-a-serious-public-healthproblem-that-affects. or RABU 13042011

http:/ / bahankuliahkesehatan.blogspot.com/

30. Shumway et al. Preterm labour, placental abruption and preterm rupture of membranes in relation to mental and verbal abuse. Journal of Maternal and Fetal Medicine 1999, 8:76-80.

31. Boy A, Salihu HM. Intimate partner violence and birth outcomes: a systematic review. International Journal Fertility and Women's Medcine 2004; 49(4): 159-64.

32. Frye, V. Examining homicide's contribution to pregnancyassociated deaths. The Journal of the American Medical Association 2001; 285(11).

33. Bullock MF, McFarlene J. The birthweight/battering connection. American Journal of Nursing 1989; 89: 1153-5.

34. Murphy CC et al. Abuse a risk factor for low birth weight?A systematic review and meta analysis. Canadian Medical Association Journal 2001; 164(11): 1567-72.

35. Woolhouse H, Gartland D, Hegarty K, Donath S, Brown S. Depressive symptoms and intimate partner violence in the 12 months after childbirth: a prospective pregnancy cohort study. British Journal of Obstetrics and Gynaecology 2011. doi: 10.1111/j.1471-0528.2011.03219.x. [Epub ahead of print
36. Abused women have special needs, Network 1998; 18. Family Health International.

37. Janssen PA et al. Intimate partner violence and adverse outcomes: a population based study. American Journal of Obstetrics and Gynaecology 2003; 188(5): 1341-7.

38. Addressing violence against women: piloting the programme guide. Ministry of Health, UNFPA and Sarvodaya Project, 2002.

39. Health sector response to gender based violence: an assessment of the Asia Pacific Region, 2010. UNFPA Asia and Pacific Regional Office.

40. Domestic violence (pregnancy). The UK NSC policy on domestic violence screening in pregnancy. UK Screening Portal. UK National Screening Committee. http:// www.screening.nhs.uk/domesticviolence-pregnancy.

41. Antenatal care routine care for the healthy pregnant woman. Clinical Guideline 2008, National Collaborating Centre for Women's and Children's Health Published by the RCOG Press at the Royal College of Obstetricians and Gynaecologists, 2008.

42. Intimate Partner Violence During Pregnancy. A Guide for Clinicians. Centers for Disease Control and Prevention. (2007). Retrieved 14.01.2011 http://www.cdc.gov/ Reproductivehealth/violence/IntimatePartnerViolence/ sld011.htm

43. Williamson KJ, Coonrod DV, Bay RC, Brady MJ, Pratap A, Wolf WL. Screening for domestic violence: practice patterns, knowledge, and attitudes of physicians in Arizona. Southern Medical Journal 2004; 97: 1049-54. 